\title{
A simple protein-based surrogate neutralization assay for SARS-CoV-2
}

Kento T. Abe, ${ }^{1,2}$ Zhijie Li, ${ }^{1}$ Reuben Samson, ${ }^{1,2}$ Payman Samavarchi-Tehrani, ${ }^{2}$ Emelissa J. Valcourt, ${ }^{3}$ Heidi Wood, ${ }^{3}$ Patrick Budylowski, ${ }^{4,5}$ Alan P. Dupuis II, ${ }^{6}$ Roxie C. Girardin, ${ }^{6}$ Bhavisha Rathod, ${ }^{2}$ Jenny H. Wang, ${ }^{2}$ Miriam Barrios-Rodiles, ${ }^{2}$ Karen Colwill, ${ }^{2}$ Allison J. McGeer, $, 7,7,9$ Samira Mubareka, ${ }^{10,11,12,13}$ Jennifer L. Gommerman, ${ }^{4}$ Yves Durocher, ${ }^{14}$ Mario Ostrowski, ${ }^{4,15,16}$ Kathleen A. McDonough, ${ }^{6,17}$ Michael A. Drebot, ${ }^{3,18}$ Steven J. Drews, ${ }^{19}$ James M. Rini,, ${ }^{1,20}$ and Anne-Claude Gingras ${ }^{1,2}$

'Department of Molecular Genetics, University of Toronto, Toronto, Ontario, Canada. ${ }^{2}$ Lunenfeld-Tanenbaum Research Institute at Mount Sinai Hospital, Sinai Health System, Toronto, Ontario, Canada. ${ }^{3}$ Zoonotic Diseases and Special Pathogens, National Microbiology Laboratory (NML), Public Health Agency of Canada, Winnipeg, Manitoba, Canada. ${ }^{4}$ Department of Immunology and ${ }^{5}$ Institute of Medical Science, University of Toronto, Toronto, Ontario, Canada. ${ }^{6}$ Wadsworth Center, New York State Department of Health, Albany, New York, USA. 'Department of Microbiology, University Health Network and Sinai Health System, Toronto, Ontario, Canada. ${ }^{8}$ Dalla Lana School of Public Health and ${ }^{9}$ Department of Laboratory Medicine and Pathobiology, University of Toronto, Toronto, Ontario, Canada. ${ }^{10}$ Department of Laboratory Medicine and Molecular Diagnostics, Division of Microbiology, Sunnybrook Health Sciences Centre, Toronto, Ontario, Canada. "Biological Sciences, Sunnybrook Research Institute, Toronto, Ontario, Canada. ${ }^{2}$ Division of Infectious Diseases, Sunnybrook Health Sciences Centre, Toronto, Ontario, Canada. ${ }^{13}$ Department of Laboratory Medicine and Pathobiology, University of Toronto, Toronto, Ontario, Canada. ${ }^{14}$ Mammalian Cell Expression, Human Health Therapeutics Research Centre, National Research Council Canada, Montréal, Quebec, Canada. ${ }^{15}$ Department of Medicine, University of Toronto, Toronto, Ontario, Canada. ${ }^{16} \mathrm{Li}$ Ka Shing Knowledge Institute, St. Michael's Hospital, Toronto, Ontario, Canada. ${ }^{17}$ Department of Biomedical Sciences, School of Public Health, University at Albany, SUNY, Albany, New York, USA. ${ }^{18}$ Department of Medical Microbiology and Infectious Disease, University of Manitoba, Manitoba, Canada. ${ }^{19}$ Canadian Blood Services, Edmonton, AB \& Department of Laboratory Medicine and Pathology, University of Alberta, Edmonton, Alberta, Canada. ${ }^{20}$ Department of Biochemistry, University of Toronto, Toronto, Ontario, Canada.

Authorship note: KTA and ZL contributed equally to this work.

Conflict of interest: SJD has acted as a content expert for respiratory viruses for Johnson \& Johnson (Janssen). Questcap provided funds to the Gingras and Mubareka labs for this project.

Copyright: (c) 2020, Abe et al. This is an open access article published under the terms of the Creative Commons Attribution 4.0 International License.

Submitted: July 16, 2020

Accepted: August 31, 2020

Published: October 2, 2020.

Reference information: JCI Insight. 2020;5(19):e142362.

https://doi.org/10.1172/jci.

insight.142362.
Most of the patients infected with severe acute respiratory syndrome coronavirus 2 (SARS-CoV-2) mount a humoral immune response to the virus within a few weeks of infection, but the duration of this response and how it correlates with clinical outcomes has not been completely characterized. Of particular importance is the identification of immune correlates of infection that would support public health decision-making on treatment approaches, vaccination strategies, and convalescent plasma therapy. While ELISA-based assays to detect and quantitate antibodies to SARS-CoV-2 in patient samples have been developed, the detection of neutralizing antibodies typically requires more demanding cell-based viral assays. Here, we present a safe and efficient protein-based assay for the detection of serum and plasma antibodies that block the interaction of the SARS-CoV-2 spike protein receptor binding domain (RBD) with its receptor, angiotensin-converting enzyme 2 (ACE2). The assay serves as a surrogate neutralization assay and is performed on the same platform and in parallel with an ELISA for the detection of antibodies against the RBD, enabling a direct comparison. The results obtained with our assay correlate with those of 2 viral-based assays, a plaque reduction neutralization test (PRNT) that uses live SARS-CoV-2 virus and a spike pseudotyped viral vector-based assay.

\section{Introduction}

The coronavirus S-protein (spike) is responsible for both receptor binding and fusion of the virus and host cell membranes. Within the spike protein, the receptor binding domain (RBD) mediates the interaction with the host cell receptor, and sequence/structural variation in the $\mathrm{RBD}$ is responsible for the receptor binding specificity shown by those coronaviruses that use host proteins as receptors (1). 
SARS-CoV-2, like SARS-CoV, uses the cell surface carboxypeptidase angiotensin-converting enzyme 2 (ACE2) as a receptor for viral entry (Figure 1A). The use of a common receptor is consistent with the fact that the 2 viruses share a high degree of sequence similarity and that their RBDs are $\sim 74 \%$ identical, though the RBD of SARS-CoV-2 binds ACE2 with higher affinity than does that of SARS-CoV (2). The spike proteins of both viruses are also both primed by the host protease, TMPRSS2, but unlike SARS-CoV-2, the spike protein of SARS-CoV does not contain a furin recognition motif that can be cleaved during viral biogenesis $(2,3)$.

The coronavirus spike protein is also a major target of the host immune system, and antibodies directed against it play a central role in host-mediated neutralization (4). Among neutralizing antibodies, those that block the interaction between viruses and their receptors represent the most common route to neutralization (5). For this reason, both the spike protein and the RBD form the basis for most of the SARS-CoV-2 vaccines currently in development. The detection and study of neutralizing antibody activity following natural infection (or vaccination) can, therefore, support research aimed at the development of novel therapeutics and vaccine candidates. It can also aid in the identification of acceptable donors for convalescent plasma therapy (6) and, more generally, to establish immune correlates of infection.

For SARS-CoV-2, viral neutralization assays are performed using either live virus (7) or viral vectors pseudotyped with the spike protein (8). However, these cell culture-based assays are challenging to implement and time-consuming to run - factors that limit scalability. The conventional plaque reduction neutralization test (PRNT) that uses live SARS-CoV-2 virus is further complicated by the need for containment level 3 (CL-3) and a specialized laboratory setup. Although the pseudotyped viral vector-based assays do not require biosafety level 3 (BSL-3) containment (8), they are complicated multistep procedures (9).

By contrast, the detection and quantitation of antigen-specific antibodies in patient samples can be easily assayed by ELISA (10). SARS-CoV-2 ELISAs are performed by immobilizing a recombinantly produced viral antigen (such as the spike trimer or RBD) (Figure 1B and Supplemental Figures 1 and 2; supplemental material available online with this article; https://doi.org/10.1172/jci.insight.142362DS1) (see Methods) onto multiwell plastic plates that are then incubated with diluted patient serum or plasma samples. The detection of antibodies that bind to the antigen involves a second incubation with enzyme-conjugated antihuman antibodies, where the enzyme is often horseradish peroxidase (HRP). This enables the detection of a color change when an HRP substrate such as 3,3',5,5'-tetramethylbenzidine (TMB) is used. In direct binding assays of this type (Figure $1 \mathrm{C}$ ), the presence of patient antibodies against the viral antigen leads to a dose-dependent increase in the signal observed.

ELISA-based profiling has been developed by multiple groups and has been used to measure the kinetics of the antibody response in patient cohorts following SARS-CoV-2 infection. In several recent studies, including ours, this has revealed the relative stability in the IgG response to the spike and RBD over several months, along with a more transient IgM and IgA response that wanes as patients convalesce (11-15). However, the levels of neutralizing antibodies are not typically measured in large cohorts over time (with a few notable exceptions, as seen in refs. 12, 15), as current assays have relatively low throughput. The relative lack of neutralizing antibody data represent a significant gap in our understanding of the immune response to SARS-CoV-2.

Here, we describe a modified ELISA-type assay that serves as a surrogate neutralization assay. It measures the presence of antibodies capable of blocking the RBD-ACE2 interaction, and like the direct binding ELISA, it is easily scaled to allow for the analysis of large patient cohorts over time. We show that the results obtained by this assay correlate with those of both the SARS-CoV-2 PRNT and a spike pseudotyped viral vector neutralization assay in a cohort of convalescent patients and on purified antibodies.

\section{Results}

We aimed to develop a simple protein-based assay to monitor the ability of antibodies, present in the serum or plasma of patients, to block the interaction between the RBD and the host receptor ACE2. To do so, we elected for an ELISA-type assay, since such assays are already widely used to detect antibodies that recognize SARS-CoV-2 antigens such as the spike trimer and its RBD. As with the standard direct ELISA, the antigen (here, the RBD or the spike trimer) is first immobilized on multi-well plates and then incubated with patient plasma or serum (Figure 1B). However, because we were interested in detecting functional antibodies that can prevent the interaction between the RBD (or spike) and ACE2, we replaced the HRP-conjugated secondary antibody used in the direct ELISA by a detection method involving human ACE2. In our assay, recombinantly expressed soluble ACE2 bearing a biotinylated C-terminal AviTag is added to the antigen-bound plate after 
A

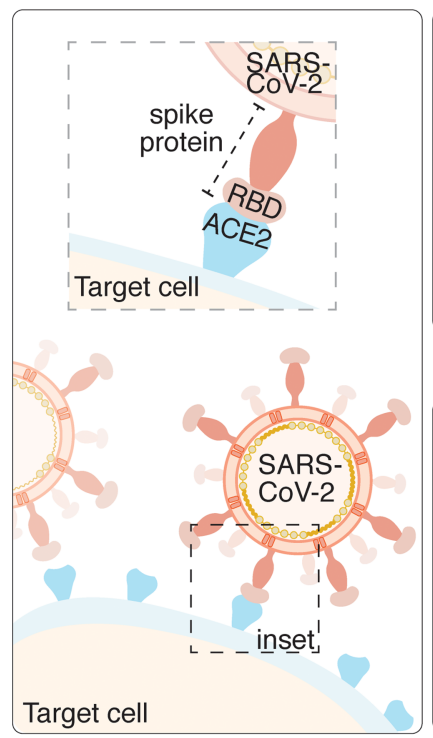

B Common ELISA setup

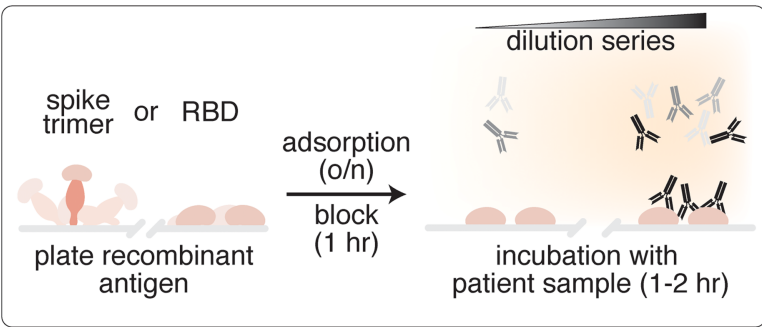

C Direct binding ELISA detection

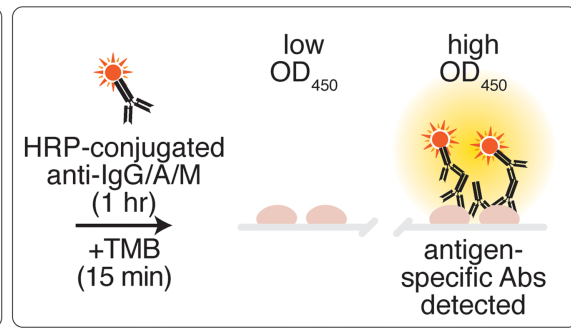

D Surrogate neutralization ELISA (snELISA) detection

biotin
tag
ACE2
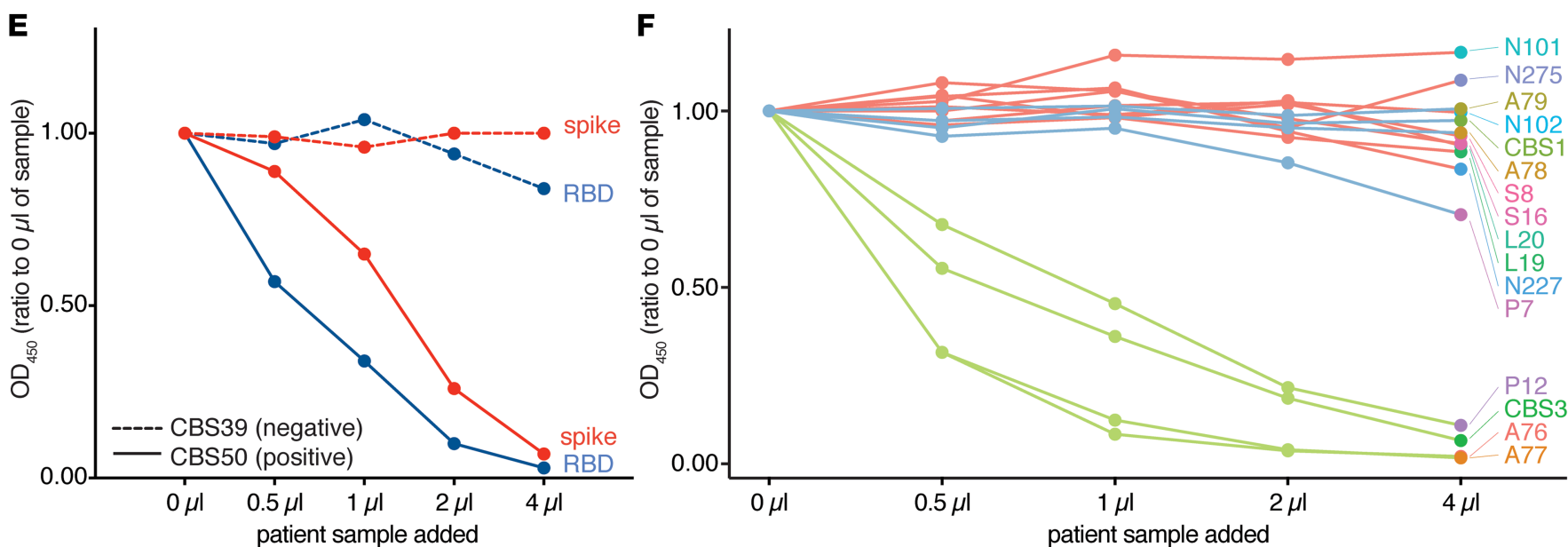

Figure 1. Establishment of a surrogate neutralization ELISA (snELISA) to monitor the spike-ACE2 interaction. (A) SARS-CoV-2 attachment to the host cell requires a direct interaction between the host cell receptor, ACE2 (blue), and the receptor binding domain (RBD) of the SARS-CoV-2 spike protein (peach). (B) Ninety-six-well plates are set up in a similar manner for both the detection of anti-SARS-CoV-2 antibodies and neutralizing antibodies. Antigens are adsorbed overnight and incubated with diluted patient serum/plasma samples, monoclonal antibodies, and other affinity reagents. Antigen-specific antibodies are colored black. (C) Principle of direct binding ELISA detection using HRP-conjugated anti-human IgG/A/M. (D) Principle of the SnELISA, which uses biotinylated ACE2 for the detection of RBD or spike epitopes that have not been blocked by neutralizing antibodies (using polyHRP-streptavidin). (E) Results of the snELISA using either the RBD or the spike trimer immobilized on the plate (see Supplemental Figures 1 and 2 for the antigen cloning, expression, and purification). The dashed lines are from a sample (CBS39) that was negative for direct RBD/spike binding, while the solid lines are from a positive sample (CBS50; see Supplemental Figure 4A for the direct binding results and Supplemental Table 1 for all $\mathrm{OD}_{450}$ values). (F) snELISA (immobilized RBD) for an expanded set of 4 positive controls with high anti-RBD signals in a single-point direct-binding ELISA (green), 8 negative samples acquired before COVID (red), and 4 samples with low anti-RBD levels (blue; Supplemental Figure 4A). The 5-point curves in $\mathbf{E}-\mathbf{F}$ were generated from 1 experiment.

the plate has been incubated with the patient plasma or serum (see Methods). Bound ACE2 is then detected by the addition of streptavidin-poly HRP and its colorimetric substrate TMB. The presence of patient antibodies that can block the RBD-ACE2 interaction leads to a dose-dependent decrease in the signal observed, and as such, we refer to it as a surrogate neutralization ELISA (snELISA; Figure 1D).

We explored 2 different versions of the assay: the configuration described above and one involving immobilized ACE2 and soluble biotinylated RBD, a configuration similar to that previously reported (16). The assay with immobilized RBD and soluble biotinylated ACE2 was more sensitive than its counterpart (Supplemental Figure 3). Moreover, with the RBD immobilized, the same overall protocol and colorimetric detection can be used for both the direct binding ELISA and the snELISA, thereby facilitating a direct comparison. 
Although the snELISA worked well with either the RBD or the spike ectodomain trimer immobilized (Figure 1E and Supplemental Figure 4A), we focused on the RBD, since it is easier to produce and provides a simple one-to-one binding interaction with ACE2. Using a small test set (Supplemental Figure 4B), we first showed that the serum/plasma from positive but not negative control patients inhibited the interaction between ACE2 and the immobilized RBD (Figure 1F). The technical reproducibility of the assay was within $5 \%-10 \%$ coefficient of variation $(\mathrm{CV})$. The total time required to perform the assay (once the plates are coated with the antigen) is 3.5-4 hours, and the assay can be performed using the same equipment and biosafety protocols as a standard ELISA.

Using both the surrogate neutralization and direct binding (with a dilution series) ELISAs, we then profiled a set of 58 serum samples acquired at the Canadian Blood Services as part of a screen for convalescent plasma therapy donors (Figure 2, A and B; Supplemental Figures 5-8; and Supplemental Table 1). With reference to the direct binding results, the snELISA showed that samples with high levels of IgG against the RBD were typically the most potent at blocking the RBD-ACE2 interaction (e.g., CBS13, which is included as a positive control). Conversely, samples lacking detectable RBD-binding antibodies were not able to block the interaction.

To more systematically evaluate the relationship between the RBD-binding antibody levels and the ability to block the RBD-ACE2 interaction (as determined by the snELISA), we calculated the AUC for both assays and plotted the RBD-binding AUC versus the snELISA AUC (Figure 2C and Supplemental Figure 9). The plot showed a clear correlation $\left(R^{2}=0.823\right)$, with the sera containing the highest RBD-binding antibody levels being the most effective at blocking the RBD-ACE2 interaction (Figure 2C; compare Figure 2B with Figure 2A; Supplemental Table 1). Nevertheless, there are samples with similar RBD-binding antibody concentrations that differ in their ability to block the RBD-ACE2 interaction (Figure 2D and Supplemental Figure 10). Differences in antibody isotype, affinities, and abundance, as well as the RBD epitopes bound, are all factors that could explain these outliers.

While it is reasonable to expect that antibodies that block the RBD-ACE2 interaction would be neutralizing, we validated this using cell-based viral infectivity and entry assays. Fifty-seven of the 58 samples analyzed by the snELISA were analyzed by PRNT, the gold standard in the field. PRNT50 is defined as the concentration of patient serum or plasma capable of reducing the formation of viral plaques by $50 \%$; PRNT90 is the concentration that reduces plaque formation by $90 \%$. As shown in Figure 3A, most of the samples displaying high values in the direct binding and snELISAs were also positive by PRNT90 (and those with low titers were negatives). Both ELISAs also gave an overall agreement with the PRNT50 titers (see Supplemental Figure 11, with a coefficient of determination of 0.6 ). We also adapted and optimized a spike-pseudotyped lentiviral-based entry assay (8), and we reprofiled the neutralization potential of a subset of samples. There was also a high correlation $\left(R^{2}\right.$ $=0.76$ ) between the snELISA results and the titers obtained with this spike-pseudotyped lentiviral-based entry assay (Figure 3B and Supplemental Figure 12). Taken together, these results indicate that our snELISA is a good surrogate neutralization assay, particularly for distinguishing between samples with high versus low neutralization activity. As such, the assay should be of value in the selection of candidate donors for convalescent plasma therapy and for monitoring immune correlates of patient outcomes. Future work will focus on providing a better understanding of the outliers observed across all assays. Indeed, rare but potent neutralizing antibodies in patient samples with low pseudovirus neutralization titers have recently been reported (17).

To assess whether our snELISA might also be of value for screening the neutralization potential of monoclonal antibodies, we tested it using a number of neutralizing and nonneutralizing monoclonal antibodies and compared the results with the results obtained with the pseudotyped lentiviral-based entry assay or cytopathic effect-reduction neutralization assay with SARS-CoV-2. The llama VHH72 monoclonal antibody (expressed as a human Fc fusion), previously shown to neutralize in a SARS-CoV-2 spike pseudotyped entry assay (18), blocked the RBD-ACE2 interaction in our snELISA and viral entry in our spike pseudotyped lentivirus assay; similar results were obtained for the Active Motif 414-1 antibody, which was isolated from a convalescent patient and was shown to be neutralizing (19) (Figure 3, C and D, and Supplemental Figure 13). In contrast, other antibodies, such as an IgG derived from the monoclonal anti-SARS1 CR3022 or a commercial antibody (HC2001) from GenScript, had a much more moderate effect in the snELISA (Supplemental Figure 14), and the GenScript antibody had no effect in the cytopathic effectreduction neutralization assay (Supplemental Figure 15). The Active Motif 414-2 antibody was previously shown to be incapable of neutralizing live SARS-CoV-2 virus $(8,19)$. In our assays, it efficiently bound the $\mathrm{RBD}$ in the direct ELISA but did not block the RBD-ACE2 interaction in the snELISA. The same antibody 
A

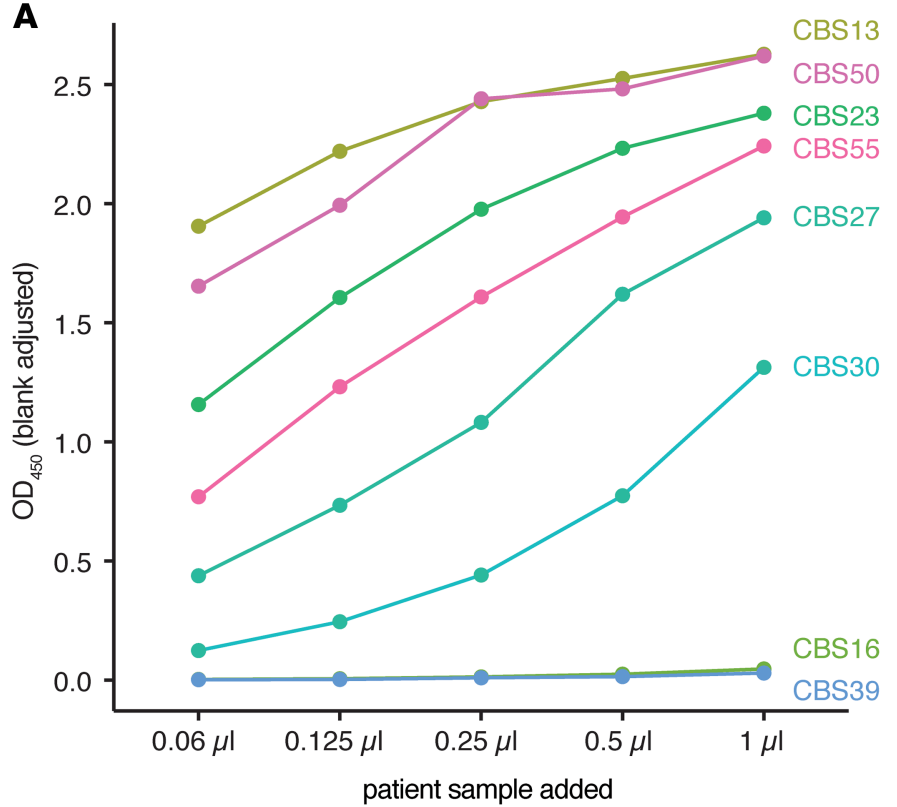

C

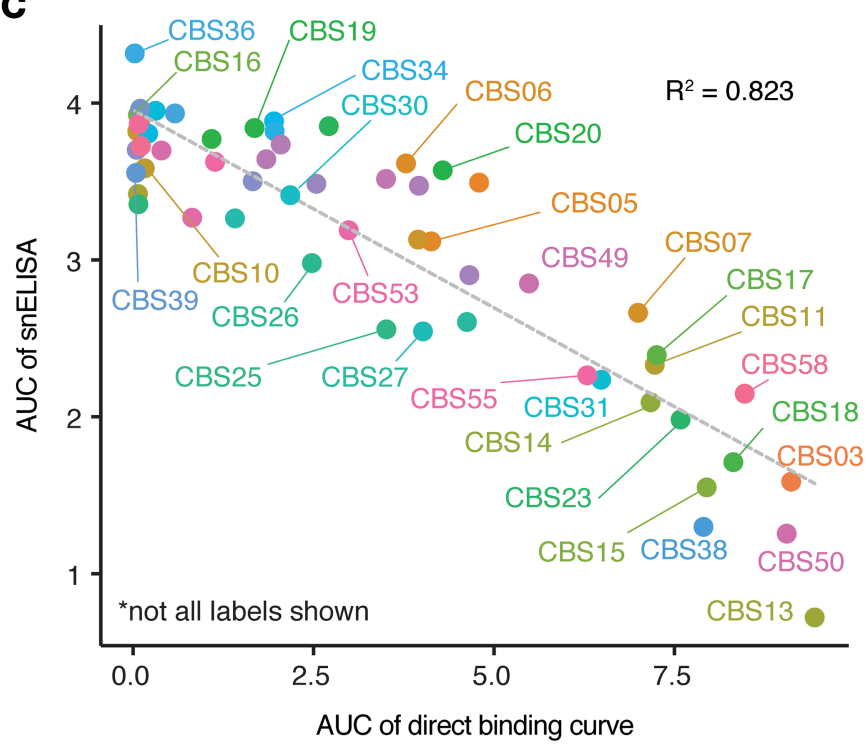

B

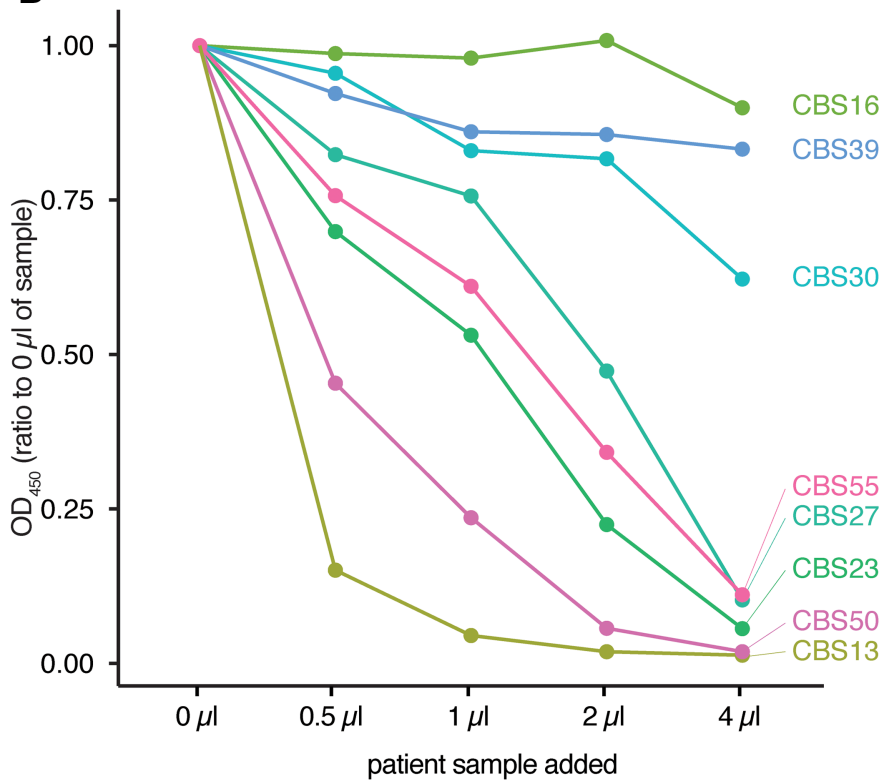

D

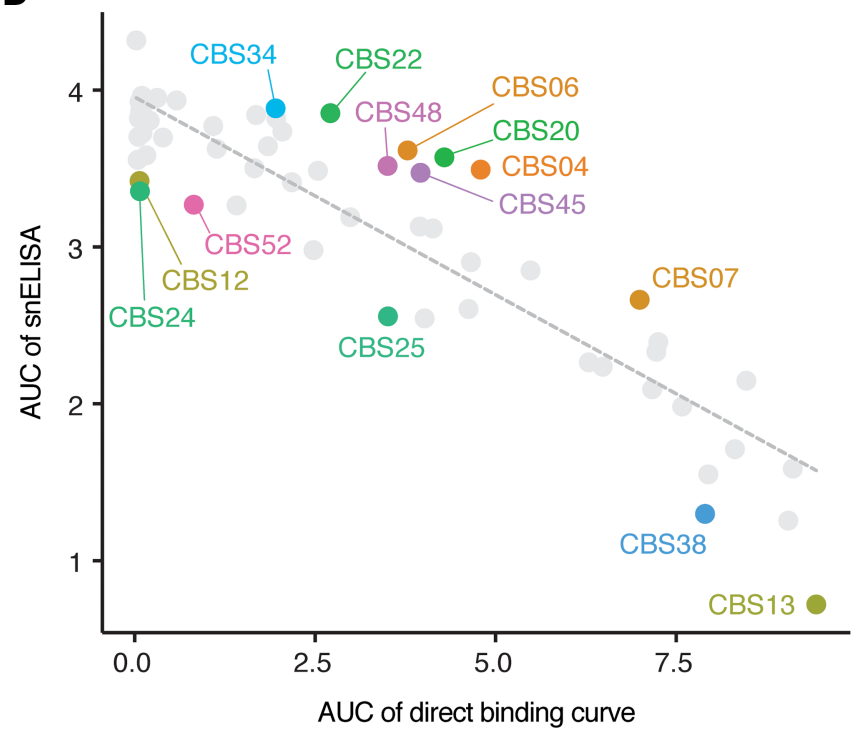

Figure 2. Application of the snELISA to a larger cohort. (A) Representative direct binding ELISAs with titrations of different samples from a patient cohort sampled by Canadian Blood Services (all 58 ELISA curves are shown in Supplemental Figure 6; see Supplemental Figure 7 for an extended titration of the most abundant samples). (B) snELISA results for the samples shown in $\mathbf{A}$; see Supplemental Figure 8 for all curves. The 5-point curves in $\mathbf{A}$ and $\mathbf{B}$ were generated from 1 experiment. (C) Correlation between the AUCs for the direct and snELISAs for all samples profiled (see an expanded view in Supplemental Figure 9). (D) Outliers in the correlation plot C were calculated using the total least squares (TLS) method; points with a TLS > 0.4 (labeled) were marked as outliers (see Supplemental Figure 10 for selected examples with side-by-side direct and snELISAs).

partially prevented entry in our lentivirus entry assay. Taken together, these observations suggest that our snELISA is a good complement to more complex cell-based assays for the discovery and screening of neutralizing monoclonal antibodies.

\section{Discussion}

In summary, we have developed a simple and safe snELISA for SARS-CoV-2. It can be readily incorporated into existing testing platforms and may be of particular value in the selection of donors for convalescent plasma therapy and as a means of monitoring the immune response to vaccination. Given that neutralizing antibody titres have recently been shown to wane fairly rapidly in some $(12-14,20)$ but not all $(15,20)$ studies, the assay may also be useful for broad serosurveillance, especially as it should be more scalable than the 
A
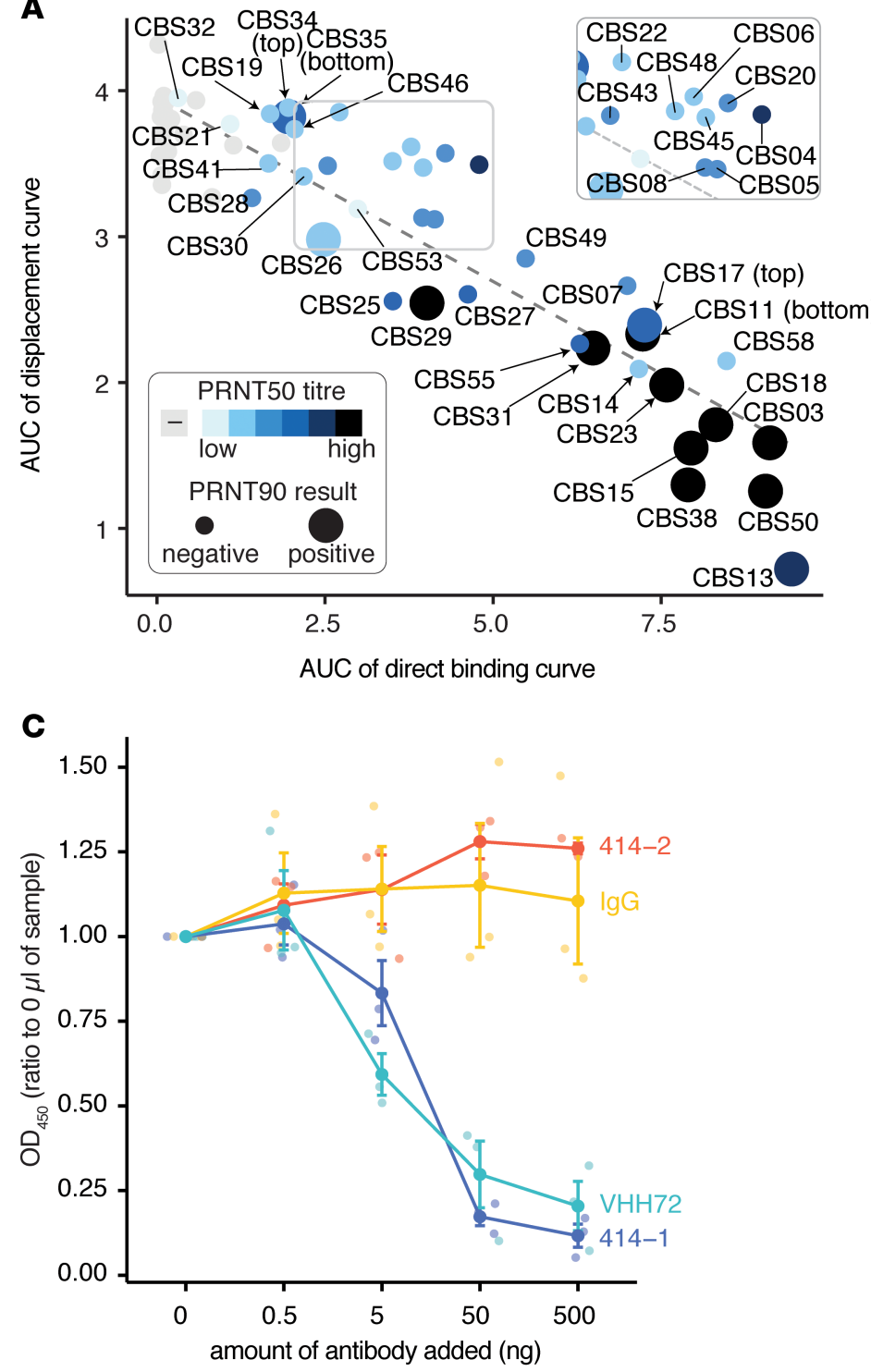

B

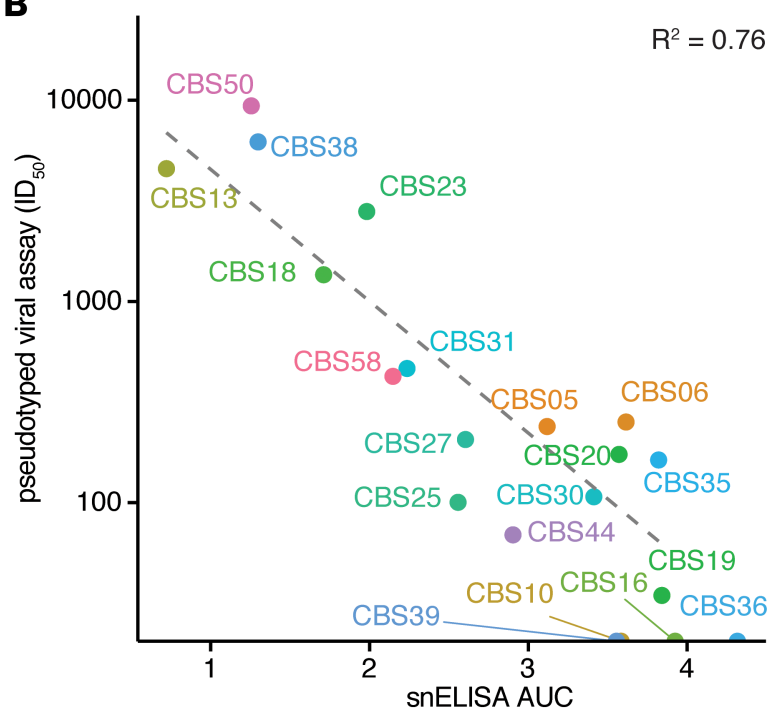

D

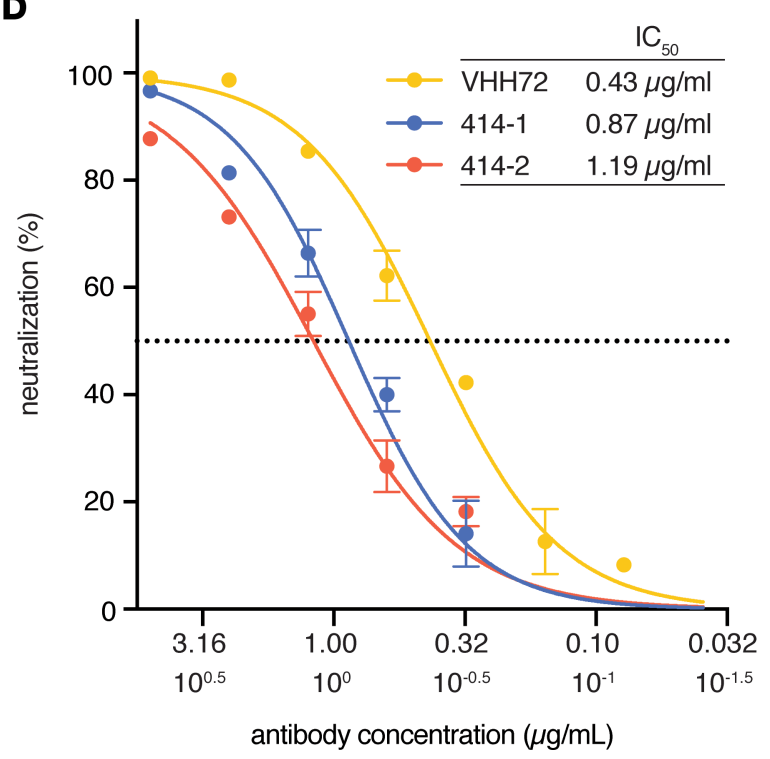

Figure 3. Validation of the snELISA results using orthogonal methods. (A) Results of the plaque reduction neutralization tests on the same samples overlaid on the AUC curves from Figure $2 C$ ( $n=57$ samples). Color coding indicates the PRNT50 titers, while negative/positive hits on the PRNT90 assay are displayed with a different-sized dot (see Supplemental Figure 11 for additional PRNT results and Supplemental Figure 12 for spike pseudotyped virus results). (B) Correlation between the lentiviral spike pseudotyped virus assay calculated $\mathrm{IC}_{50}$ values and the AUC results from the snELISA. (C and $\mathbf{D}$ ) Assessment of the ability of monoclonal or affinity reagents to block the interaction between ACE2 and the RBD in the snELISA (C) or the lentiviral spike pseudotyped assay (D) (see Supplemental Figure 13-16 for the direct binding and viral neutralization assays and for additional reagents tested). Values for C were generated from 3 independent experiments, and underlayed points have been placed along the $x$ axis. Values for $\mathbf{D}$ were obtained in 1 experiment in technical triplicate. Data are presented as mean \pm SEM.

approaches requiring viral infection assays. When coupled with epidemiological studies, it might also be used to assess the risk of infection/reinfection. We also note that the optimized conditions used here for the direct RBD-binding ELISA are similar to those reported in ref. 10 using RBD-expression constructs that have been widely distributed. Their RBD can be obtained from BEI Resources (10), and we found that it generates similar results when used with our biotinylated ACE2 in the snELISA (Supplemental Figure 16). This should further facilitate the broad implementation of our assay across multiple laboratories.

There are limitations to the assay, however, that need to be acknowledged. First, the snELISA is limited to the detection of neutralizing antibodies that function by blocking the interaction between the RBD and ACE2. While by no means dominant, examples of antibodies that neutralize by other mechanisms are 
beginning to emerge (21-23). The snELISA, in conjunction with a neutralization assay, could be used to identify further such examples. As with those identified in this work, the outliers (e.g., those with high viral neutralization titers but low snELISA levels) provide a starting point for further work aimed at understanding the mechanisms of antibody-mediated neutralization.

Another limitation of our approach is that the current assay cannot directly map the epitopes targeted by the various antibodies. Undoubtedly, the antibodies detected by the snELISA bind to different sites on the RBD, a suggestion supported by the structures of neutralizing antibody fragment antigen binding (Fabs) in complex with the SARS-CoV-2 RBD. In one example, 2 different neutralizing antibodies that bind to different epitopes on the RBD were found to synergistically mediate viral neutralization (24). While, in the current study, we simply wanted to provide evidence of antibodies that could block the RBD-ACE2 interaction, the snELISA could be adapted to provide information on the site of antibody binding. As recently shown, a series of structure-guided point mutants in the RBD could be used to infer where on the RBD the antibodies are binding (25). This type of approach would likely be more important in the characterization of monoclonal antibodies, such as those presented in Figure 3C, and would set the stage for in-depth biophysical and structural studies.

While the direct binding ELISA described here employed an anti-IgG secondary antibody (the predominant isotype in convalescent serum), we note that the snELISA measures the ability of any antibody isotype (or even antibodies from different species or any other molecule) to block the RDB-ACE2 interaction. In this regard, it is similar to that of a viral-based neutralization assay. While we have not performed a detailed analysis, we did show that single-point direct binding ELISAs performed for IgM, and to a lesser extent IgA, are also correlated with the results obtained by the snELISA (Supplemental Figure 17). The safety and simplicity of the snELISA should make it a valuable addition to the arsenal of assays for monitoring the immune response to SARS-CoV-2 infection.

\section{Methods}

\section{Serum and plasma samples}

Canadian Blood Services donors. Specimens-only serum donations were collected from individuals who were classified as having 1 or more of the following criteria: (a) self-declared evidence of a SARS-CoV-2positive nucleic acid test, (b) a declaration of having been a close contact of a COVID-19 case, (c) a travel history and clinical presentation compatible with COVID-19, and (d) signs and symptoms compatible with COVID-19. Collections occurred 2 weeks or more after cessation of clinical symptoms. Serum specimens were processed and frozen at $-80^{\circ} \mathrm{C}$ until shipment on dry ice to the testing laboratory.

Other samples for assay development. Negative control serum samples from patients enrolled in cancer studies before COVID-19 (before November 2019; REB studies 01-0138-U and 01-0347-U, Mount Sinai Hospital) and archived frozen in the LTRI Biobank were retrieved, thawed, aliquoted, and transferred on ice to the research lab for viral inactivation. Alternatively, samples from previous studies of the immune system or systemic lupus acquired before November 2019 (REB studies 31593, University of Toronto, 05-0869, University Health Network, a gift from Joan Wither, University Health Network) were transferred to the lab on dry ice. Positive controls for assay development were either convalescent plasma or serum from COVID-19 patients (confirmed by PCR; in- and out-patients) acquired in south-central Ontario in 2020 (REB studies 20-044, Unity Health Network (Toronto, Canada), 02-0118-U/05-0016-C, Mount Sinai Hospital). Aliquots of these samples were transferred to the lab on dry ice. Only those samples with high levels of RBD-binding antibodies in single-point ELISAs were considered positives for the development of the snELISA. Samples with low levels of RBD-binding antibodies were reclassified as negative.

For all ELISAs, inactivation of potential infectious viruses in plasma or serum was performed by incubation with Triton X-100 to a final concentration of $1 \%$ for 1 hour before use (26). For the pseudotyped lentiviral assays, the serum was heat inactivated for 1 hour at $56^{\circ} \mathrm{C}(10)$.

\section{Expression system for protein purification}

The expression plasmid generated is a derivative of those previously reported in our piggyBac transposonbased mammalian cell expression system (27). Two versions of the plasmid were constructed: one contains the CMV promoter (PB-CMV) and the other the TRE promoter (PB-TRE). The vectors are otherwise identical and can be used to generate stable cell lines for constitutive or inducible protein expression. The protein cloning 
region contains several optional elements separated by restriction sites as follows: an N-terminal human cystatin-S secretion signal, the protein of interest, a foldon trimerization motif (28), a 6xHis purification tag, and an AviTag biotinylation motif (29) (Supplemental Figure 1). A woodchuck hepatitis virus posttranscriptional regulatory element (WPRE) follows the ORF to facilitate nuclear export of the mRNA. A pair of piggyBac transposon terminal repeats flank the expression cassette and an attenuated puromycin resistance marker (BioShop Canada Inc., PUR333), thereby allowing for the generation of stable cell lines using the piggyBac transposase.

\section{Expression constructs for protein purification}

The human codon optimized cDNA of the SARS-CoV-2 spike protein (MC_0101081) was purchased from GenScript. The human ACE2 cDNA was derived from MGC clone 47598. To stabilize the soluble spike ectodomain trimer, 2 regions of the spike protein were mutated. Residues 682-685 (RRAR) were mutated to SSAS to remove the furin cleavage site, and residues 986-987 (KV) were each mutated to a proline residue to stabilize the prefusion form as previously described (30). The soluble spike protein ectodomain construct includes residues 1-1211 (MFVF...QYIK), followed by the foldon trimerization motif, a 6xHis tag, and an AviTag. Both the SARS-CoV-2 RBD and the human ACE2 constructs are preceded by the human cystatin-S secretion signal and followed by the $6 x$ His and AviTag. The RBD and ACE2 constructs contain residues 328-528 (RFPN...CGPK) and 19-615 (STIE...PYAD), respectively.

The cDNA of the human CR3022 Fab was synthesized by GenScript based on its previously reported sequence (31). The light chain and heavy chains were individually cloned into the PB-TRE expression plasmid. For Fab production, a $6 x$ His tag was added to the C-terminal end of the Fab heavy chain. An IgG form was generated by fusing the human IgG1 Fc coding sequence to the C-terminal end of the Fab heavy chain.

\section{Large-scale transient transfection}

FreeStyle 293-F suspension cells (Thermo Fisher Scientific, R79007) were grown in shaker flasks (125 rpm) in FreeStyle 293 expression medium (Thermo Fisher Scientific, 12338018) in a humidified $37^{\circ} \mathrm{C}$ incubator filled with $3 \%(\mathrm{v} / \mathrm{v}) \mathrm{CO}_{2}$. The cell density and viability were monitored by manual counting using a hemocytometer and trypan blue staining. For transfection, cells of $>90 \%$ viability were counted and seeded at a density of approximately $1 \times 10^{6}$ cells $/ \mathrm{mL}$ into $300 \mathrm{~mL}$ FreeStyle 293 medium supplemented with $1 \mu \mathrm{g} / \mathrm{mL}$ aprotinin (BioShop Canada Inc., APR600). The PB-CMV plasmid DNA $(300 \mu \mathrm{g})$ and 293 fectin $(400 \mu \mathrm{L}$; Thermo Fisher Scientific, 12347019) were each added to separate tubes containing $15 \mathrm{~mL}$ of Opti-MEM medium (Thermo Fisher Scientific, 31985062). The 2 solutions were then mixed and incubated for 5 minutes before being added to the $300 \mathrm{~mL}$ cell culture. Two days after transfection, the $300 \mathrm{~mL}$ culture was expanded into three $1 \mathrm{~L}$ shaker flasks each containing $300 \mathrm{~mL}$ of culture medium. Protein expression was continued for an additional 4 days.

\section{Stable cell line generation}

FreeStyle 293-F cells or a GnT1-KO FreeStyle 293-F cell line were used for generating stable cell lines. Approximately $1 \times 10^{6}$ cells were added to each well of a 6-well plate in $2 \mathrm{~mL}$ FreeStyle 293 medium. A total of $2 \mu \mathrm{g}$ of the PB-TRE plasmid encoding the protein of interest, $0.5 \mu \mathrm{g}$ of the PB-rtTA-neomycin helper plasmid (27), and $0.5 \mu \mathrm{g}$ of the PBase expression plasmid pCyL43 (32) were cotransfected in each well using lipofectamine 2000 (Thermo Fisher Scientific, 11668027) following the manufacturer's instructions. Three days after transfection, the cells were transferred to $10 \mathrm{~cm}$ dishes containing FreeStyle 293 medium supplemented with 10\% FBS (Wisent Bio Products, 080-150), $2 \mu \mathrm{g} / \mathrm{mL}$ puromycin, and $200 \mu \mathrm{g} /$ mL G418 (Thermo Fisher Scientific, 11811031). Selection was continued for approximately 2 weeks.

The stable cells were scaled up in $1 \mathrm{~L}$ shaker flasks containing $300 \mathrm{~mL}$ FreeStyle 293 medium without supplements. When the cell densities reached approximately $1 \times 10^{6}$ cells $/ \mathrm{mL}, 1 \mu \mathrm{g} / \mathrm{mL}$ doxycycline (MilliporeSigma, D3447), and $1 \mu \mathrm{g} / \mathrm{mL}$ Aprotinin were added to initiate protein expression. During the expression phase, $150 \mathrm{~mL}$ of the medium was removed, and fresh medium added every other day.

\section{Protein purification}

The harvested expression medium was centrifuged at $10,000 \mathrm{~g}$ for 30 minutes at $10^{\circ} \mathrm{C}$ to remove the cells and debris. For the $6 \mathrm{xHis}$ tagged proteins, the clarified media were passed through an Ni-NTA column (Qiagen, 30230). For the spike ectodomain, $3 \mathrm{~mL}$ of Ni-NTA resin was used for each liter of medium. For the RBD, ACE2, and CR3022 Fab, $8 \mathrm{~mL}$ of Ni-NTA resin was used for each liter of medium. The Ni-NTA resin was washed with 20 column volumes of phosphate buffered saline (PBS), followed by 3-5 column volumes of 
PBS containing $10 \mathrm{mM}$ imidazole. The protein was eluted with PBS containing $300 \mathrm{mM}$ imidazole (Bio Basic, IB0277) and 0.1\% (v/v) protease inhibitor cocktail (MilliporeSigma, P-8849). For the CR3022 antibody, the harvested medium was incubated with rProtein A Sepharose FF resin (GE Healthcare, 17127903). The resin was then washed with 20 column volumes of PBS, and the antibody was eluted with $50 \mathrm{mM}$ glycine, $\mathrm{pH}$ 3.0, containing $150 \mathrm{mM} \mathrm{NaCl}$. The acid-eluted antibody was immediately neutralized by the addition of $1 / 20$ volume of $1 \mathrm{M}$ Tris, $\mathrm{pH}$ 8.5. Protease inhibitor cocktail was also added to a final concentration of $0.1 \%(\mathrm{v} / \mathrm{v})$. The approximate purified yields of the various proteins are as follows: RBD, $70 \mathrm{mg} / \mathrm{L}$; spike trimer, $3 \mathrm{mg} / \mathrm{L}$; ACE2, $50 \mathrm{mg} / \mathrm{L}$; CR3022 Fab, $80 \mathrm{mg} / \mathrm{L}$; and CR3022 IgG, $20 \mathrm{mg} / \mathrm{L}$.

The protein samples were stored in $40 \%$ glycerol at $-12^{\circ} \mathrm{C}$. Shortly before use, the glycerol stocks were further purified using size-exclusion chromatography. For the RBD, ACE2, and CR3022 Fab/IgG, a Superdex 200 Increase (GE Healthcare, 28990944) column was used. For the spike ectodomain, a Superose 6 Increase (GE Healthcare, 29091596) column was used (Supplemental Figure 2).

\section{Site-specific biotinylation of the AviTag-containing proteins}

Each biotinylation reaction contained $200 \mu \mathrm{M}$ biotin, $500 \mu \mathrm{M}$ ATP (MilliporeSigma, Cat \# A2383), 500 $\mu \mathrm{M} \mathrm{MgCl}_{2}, 30 \mu \mathrm{g} / \mathrm{mL}$ BirA (produced from E. coli; a gift from Walid Houry, University of Toronto), $0.1 \%$ (v/v) protease inhibitor cocktail, and no more than $100 \mu \mathrm{M}$ of the protein-AviTag substrate. The mixture was incubated at $30^{\circ} \mathrm{C}$ for 2 hours, followed by size-exclusion chromatography to remove unreacted biotin (BioShop Canada Inc., BIO302). For the RBD, the degree of biotinylation was assessed using a band-shift assay. A total of $5 \mu \mathrm{g}$ of the biotinylated RBD was heated to $95^{\circ} \mathrm{C}$ for 30 seconds in SDS-PAGE loading buffer (containing $2 \%$ SDS, $50 \mathrm{mM} \mathrm{DTT}$ ); after cooling, $1 \mu \mathrm{L}$ of a $5 \mathrm{mg} / \mathrm{mL}$ streptavidin solution was added. The mixture was then analyzed by SDS-PAGE to assess the formation of the RBD-streptavidin complex (Supplemental Figure 2).

\section{Production of the $\mathrm{VHH72}$ recombinant antibody}

The llama single domain antibody VHH72 sequence (PDB entry 6WAQ 1) was obtained from Wrapp et al. (18). A cDNA encoding VHH72 fused to an ADCC-attenuated human IgG1 Fc domain (hFc1X7, from patent US 2019352 383A1) was codon optimized for expression in CHO cells, synthesized by GenScript, and cloned into the pTT5 plasmid (33). The pTT5-VHH72hFc1X7 plasmid was transiently expressed in $\mathrm{CHO}^{55 \mathrm{E} 1}$ cells (34) using PEI MAX transfection reagent (Polysciences) and a slightly modified protocol as described previously (35). The cell culture was harvested at day 7 after transfection, centrifuged 20 minutes at $3000 \mathrm{~g}$ at room temperature, and filter sterilized using a $0.22 \mu \mathrm{m}$ membrane vacuum filter (Express PLUS, MilliporeSigma). Filtered supernatant was loaded on a $5 \mathrm{~mL}$ MabSelect SuRe column (GE Healthcare) equilibrated in PBS. The column was washed with PBS, and the antibody eluted with $100 \mathrm{mM}$ citrate buffer $\mathrm{pH}$ 3.6. The fractions containing the antibody were pooled, and elution buffer was exchanged for PBS using NAP-25 columns (GE Healthcare). Purified VHH72hFc1X7 in PBS was quantified by absorbance at $280 \mathrm{~nm}$ using a NanoDrop spectrophotometer (Thermo Fisher Scientific) and the calculated extinction coefficient of the protein. Overall volumetric yield after protein A purification was $275 \mathrm{mg} / \mathrm{L}$. The purified protein was analyzed by analytical size-exclusion ultra high-performance liquid chromatography coupled to a MALS detector and eluted as a major (>98\% integrated area) symmetrical peak of $102 \mathrm{kDa}$ with less than $2 \%$ aggregates (not shown).

\section{Sources of other commercial proteins and recombinant antibodies}

An alternative source for RBD was BEI Resources NR-52306 (contributors F. Krammer, F. Amanat, S. Strohmeier; Icahn School of Medicine, Mount Sinai, New York, USA; lot 7034437). Commercial antibodies tested also included a human IgG chimeric antibody from GenScript (SARS-CoV-2 spike S1 antibody, HC2001; GenScript, A02038) and 2 SARS-CoV-2 spike antibodies from Active Motif (AM002414, 91349; AM001414, 91361).

\section{Direct ELISA for the identification of antibodies to the RBD}

Manual single-point ELISAs in 96-well format. For the manual single-point ELISAs in 96-well format, concentrations and incubation times were optimized to maximize the separation between anti-RBD levels in convalescent plasma or serum from that of pre-COVID-era banked serum while maintaining the required levels of antigens as low as possible. A total of $1 \mu \mathrm{L}$ of serum or plasma was used for the detection of 
antibodies on 96-well plates coated with $75 \mathrm{ng} /$ well of recombinant purified RBD. Single-point ELISAs are expressed as ratios to a positive control convalescent plasma sample.

Multipoint ELISAs. For the multipoint ELISAs, the RBD amount was fixed to $100 \mathrm{ng} /$ well to match the design of the snELISA, and 2-fold serial dilutions of the serum or plasma sample from $1 \mu \mathrm{L}$ to $0.06 \mu \mathrm{L}$ were employed.

Both cases. In both cases, the RBD antigen (diluted to $2 \mu \mathrm{g} / \mathrm{mL}$ in PBS) was first adsorbed to 96-well clear Immulon 4 HBX plates (Thermo Fisher Scientific, 3855) in PBS overnight at $4^{\circ} \mathrm{C}$ and then washed 3 times with $200 \mu \mathrm{L}$ PBS plus 0.1\% Tween-20 (PBS-T; MilliporeSigma). Plates were blocked for 1 hour at room temperature with $200 \mu \mathrm{L}$ 5\% Blocker BLOTTO (Thermo Fisher Scientific, 37530) and washed 3 times with $200 \mu \mathrm{L}$ PBS-T. In the single-point ELISAs, plate blocking was performed with $3 \% \mathrm{w} / \mathrm{v}$ milk powder (BioShop Canada Inc., ALB005.250, lot 9H61718) in PBS for 1-2 hours.

Patient samples (pretreated with 1\% final Triton X-100 for viral inactivation) diluted in PBS-T containing $1 \% \mathrm{w} / \mathrm{v}$ milk powder (1:50 for the single-point ELISA) were then added to the plates and incubated for 2 hours at room temperature ( $50 \mu \mathrm{L}$ total volume); technical duplicates were performed unless otherwise indicated. A chimeric human anti-spike antibody (SARS-CoV-2 spike S1 Antibody, HC2001; GenScript, A02038) was added to a set of wells on each plate as a serial dilution (1:5,000$1: 80,000$ or $10-0.63 \mathrm{ng}$ per well in 4 steps) to enable cross-plate comparisons. Positive (convalescent plasma from a single patient) and negative controls (pre-COVID-era banked serum) were also added to each plate, at $1 \mu \mathrm{L}$.

Wells were washed 3 times with $200 \mu \mathrm{L}$ PBS-T. Goat anti-human anti-IgG (goat anti-human IgG Fcy HRP, Jackson Immunoresearch, 109-035-098) at a 1:60,000 dilution (0.67 ng/well) in 1\% BLOTTO was added and incubated for 1 hour. Wells were washed 3 times with $200 \mu \mathrm{L}$ PBS-T, and $50 \mu \mathrm{L}$ of 1-Step Ultra TMB-ELISA Substrate Solution (Thermo Fisher Scientific, 34029) was added for 15 minutes at room temperature. The reaction was quenched with $50 \mu \mathrm{L}$ stop solution containing $0.16 \mathrm{~N}$ sulfuric acid (Thermo Fisher Scientific, N600). The plates were read in a spectrophotometer (BioTek Instruments Inc., Cytation 3) at $450 \mathrm{~nm}$. For all ELISA-based assays, raw OD values had blank values subtracted before analysis. For the single-point direct binding assay, the average CV across CBS samples is 3.3\% (mean) and 1.8\% (median) (Supplemental Table 1). For single-point assays, all data were normalized to the positive serum control (single point) on each plate and expressed as a ratio to this control. For the multipoint dose responses, blank-adjusted reads were used.

Variations. Variations to this protocol included the following. (a) Replacement of the RBD on the plate by the BEI Resources NR-52306. The assay was set up identically to and in parallel with our in-house produced RBD (Supplemental Figure 16). (b) Replacement of RBD (100 ng) on plate by the spike trimer purified above (667 ng) (Supplemental Figure 3). (c) Performing the single-point ELISAs using an automated platform with chemiluminescent detection for anti-IgG, -IgA, and -IgM, exactly as described in (11) (Supplemental Figure 17).

snELISA for the identification of neutralizing antibodies

Our final optimized snELISA used 100 ng immobilized recombinant RBD on 96-well Immulon HBX plates incubated overnight at $4^{\circ} \mathrm{C}(2 \mu \mathrm{g} / \mathrm{mL})$. All volumes added to the well were $50 \mu \mathrm{L}$, unless specified otherwise. Plates were washed 3 times with $200 \mu \mathrm{L}$ PBS-T and blocked for 1-1.5 hours at room temperature with $200 \mu \mathrm{L} \mathrm{3 \%} \mathrm{BSA} \mathrm{(BioShop} \mathrm{Canada} \mathrm{Inc.,} \mathrm{SKI400.1,} \mathrm{lot} \mathrm{9H61850).} \mathrm{After} \mathrm{washing} \mathrm{as}$ above, a 4-step, 2-fold serial dilution series of patient serum or plasma ( $0.5-4 \mu \mathrm{L}$ of sample) was incubated for 1 hour. The wells were washed as above and incubated with 50 ng biotinylated recombinant ACE2 for 1 hour. After washing as above, the wells were incubated with $44 \mathrm{ng}$ streptavidin-peroxidase polymer (MilliporeSigma, S2438). The resultant signal was developed and quantified with TMB in an identical manner to the direct ELISAs. Due to day-to-day variation in signal, all $\mathrm{OD}_{450}$ values are normalized to the $\mathrm{OD}_{450}$ of the well where no patient serum/antibody was added for each sample. All values are expressed in this ratio space.

Variations. Variations of this protocol included using a different source of RBD (BEI Resources, NR-52306) and using spike trimer as shown above (670 ng/well) (Figure 1C and Supplemental Figure 16). Another variation of the assay was to bind nonbiotinylated ACE2 to the plate (100 ng) and to use biotinylated RBD (50 ng) for detection (Supplemental Figure 3). 


\section{Viral neutralization assays}

Neutralization assays on the Canadian Blood Services samples used in Figure 2 were performed by 2 independent laboratories, the NML of the Public Health Agency of Canada, and the Wadsworth Center, New York State Department of Health. The cytopathic effect-reduction neutralization assay on the recombinant GenScript antibody was performed in Toronto.

For the PRNT assay at NML, SARS-CoV-2 (Canada/ON_ON-VIDO-01-2/2020, EPI_ISL_42517) stocks were titrated (7) for use in a PRNT adapted from a previously described method for SARS-CoV (36). Briefly, serological specimens were diluted 2-fold from 1:20 to 1:640 in DMEM supplemented with 2\% FBS and incubated with $50 \mathrm{PFU}$ of SARS-CoV-2 at $37^{\circ} \mathrm{C}$ and $5 \% \mathrm{CO}_{2}$ for 1 hour. The sera-virus mixtures were added to 12 -well plates containing Vero E6 cells at $100 \%$ confluence, followed by incubation at $37^{\circ} \mathrm{C}$ and $5 \% \mathrm{CO}_{2}$ for 1 hour. After adsorption, a liquid overlay composed of $1.5 \%$ carboxymethylcellulose diluted in MEM, supplemented with 4\% FBS, L-glutamine, nonessential amino acids, and sodium bicarbonate, was added to each well; the plates were incubated at $37^{\circ} \mathrm{C}$ and $5 \% \mathrm{CO}_{2}$ for 72 hours. The liquid overlay was removed, and the cells were fixed with $10 \%$ neutral-buffered formalin for 1 hour at room temperature. The monolayers were stained with $0.5 \%$ crystal violet for 10 minutes and washed with $20 \%$ ethanol. Plaques were enumerated and compared with controls. The highest serum dilution resulting in $50 \%$ and $90 \%$ reduction in plaques compared with controls were defined as the PRNT50 and PRNT90 endpoint titers, respectively. PRNT50 titers $\geq 1: 160$ and PRNT90 titers $\geq 1: 20$ were considered positive.

For the PRNT assay at Wadsworth, the assay for the detection of SARS-CoV-2 neutralizing antibodies was a modified version of previously described methods (37-39). Patient sera and SARS-CoV-2 (USA/WA-1/2020, BEI Resources, NR-52281) were diluted in Vero E6 cell culture maintenance medium (EMEM, 2\% heat-inactivated FBS, $200 \mathrm{U} / \mathrm{mL}$ penicillin G, $200 \mathrm{U} / \mathrm{mL}$ streptomycin). Patient samples were serially diluted 1:10-1:320 and mixed with an equal volume of virus containing $150 \mathrm{PFUs}$. Virus and serum mixtures were incubated at $37^{\circ} \mathrm{C}$ and $5 \% \mathrm{CO}_{2}$ for 1 hour. Following the initial incubation, $0.1 \mathrm{~mL}$ of each dilution was plated in a single well of a 6-well plate containing confluent monolayers of Vero E6 cells (ATCC, CRL-1586) and allowed to adsorb for 1 hour at $37^{\circ} \mathrm{C}$ and $5 \% \mathrm{CO}_{2}$. Following adsorption, cell cultures were overlaid with $0.6 \%$ agar in cell culture medium and returned to the incubator. At 2 days after infection, a second overlay containing $0.2 \%$ neutral red was added. Monolayers were inspected for 2 days, and plaques were counted. Antibody titers were reported as the inverse of the serum dilution resulting in $50 \%$ (PRNT50) and 90\% (PRNT90) reduction in plaques as compared with the virus inoculum control.

For the cytopathic effect-reduction neutralization assay in Toronto, $200 \mu \mathrm{L}$ of $0.2 \times 10^{6}$ VeroE6 cells $/ \mathrm{mL}$ were seeded into a 96 -well flat-bottom plate to adhere overnight. All plasma and serum samples were heat inactivated at $56^{\circ} \mathrm{C}$ for 30 minutes. In a separate 96 -well plate, the serum, plasma, or antibody $(1 \mu \mathrm{g} / \mathrm{mL})$ samples were serially diluted 2-fold 8 times in serum-free DMEM starting from a dilution of 1:20 to 1:2560 in a volume of $25 \mu \mathrm{L}$. To all wells, $25 \mu \mathrm{L}$ of SARS-CoV-2 SB2 Clone 1 was added, ensuring that each well had a dose of 100 issue culture infectious dose (TCID). For the cell control, $50 \mu \mathrm{L}$ of serum-free DMEM was added. For the virus control, $25 \mu \mathrm{L}$ of SARS-CoV-2 SB2 Clone 1 was added with a dose of 100 TCID and topped off with $25 \mu \mathrm{L}$ of serum free DMEM. The plate was incubated for 1 hour at $37^{\circ} \mathrm{C}, 5 \% \mathrm{CO}_{2}$ with shaking every 15 minutes. After incubation, all the media from the VeroE6 culture were removed, and the full $50 \mu \mathrm{L}$ of serum/SARS-CoV-2 coculture was layered on the cells. The plate was again incubated for 1 hour at $37^{\circ} \mathrm{C}, 5 \% \mathrm{CO}_{2}$, with shaking every 15 minutes. After the incubation, the inoculum was removed, and $200 \mu \mathrm{L}$ of DMEM containing $2 \%$ FBS was added. The plate was incubated for 5 days and cytopathic effect was tracked.

\section{Lentiviral spike pseudotyping assay}

The assay was established using constructs previously described (8) (constructs obtained through a gift from Jesse Bloom and Katharine Crawford, Fred Hutchison Cancer Research Centre, Seattle, Washington, USA, and now available through BEI Resources) and optimized in-house. Major changes to the reported protocol included: (a) use of a second-generation psPAX2 (Addgene, 12260) lentivirus packaging system instead of the third-generation system used by the Bloom lab, (b) production of spike pseudotyped virus-like particles (VLPs) at $33^{\circ} \mathrm{C}$, (c) a neutralization assay plate layout that increases throughput, (d) adjustments to the luciferase protocol to minimize variability in readings, and (e) use of a cell line that coexpresses ACE2 and TMPRSS2. To generate this cell line, entry vectors for ACE2 and TMPRSS2 coding sequences were cloned into pLenti CMV Puro DEST (Addgene, 17452) and pLenti CMV Hygro DEST (Addgene, 17454), respectively. The resulting 
transfer vectors were used to generate lentivirus via the second-generation psPAX2 and VSV-G (Addgene, 8454). HEK293T cells were transduced with ACE2 lentivirus at an MOI < 1 and selected with puromycin (1 $\mu \mathrm{g} / \mathrm{mL}$ ) to generate a stable population. These cells were subsequently transduced with TMPRSS2 lentivirus and selected with hygromycin $(200 \mu \mathrm{g} / \mathrm{mL})$ in a similar fashion.

For VLP generation, HEK293T cells were transiently cotransfected in a 6-well-plate format containing $2 \mathrm{~mL}$ growth medium (10\% FBS, 1\% penicillin/streptomycin [Pen/Strep] in DMEM) with $1.3 \mu \mathrm{g}$ psPAX2, $1.3 \mu \mathrm{g}$ pHAGE-CMV-Luc2-IRES-ZsGreen-W (BEI, NR-52516; a gift from Jesse Bloom and Katharine Crawford; lentiviral backbone plasmid that uses a CMV promoter to express luciferase followed by an IRES and ZsGreen), and $0.4 \mu \mathrm{g}$ HDM-IDTSpike-fixK (BEI, NR-52514; a gift from Jesse Bloom and Katharine Crawford; expressed under a CMV promoter a codon-optimized Wuhan-Hu-1 spike; GenBank, NC_045512) using $8 \mu \mathrm{L}$ JetPrime (Polyplus-transfection SA, 114-01)in $500 \mu \mathrm{L}$ JetPrime buffer. After 8 hours of transfection, the medium was replaced by $3 \mathrm{~mL}$ of DMEM containing $5 \%$ heat-inactivated FBS and $1 \%$ Pen/Strep, and the cells were incubated for 16 hours at $37^{\circ} \mathrm{C}$ and $5 \% \mathrm{CO}_{2}$; they were then transferred to $33^{\circ} \mathrm{C}$ and $5 \%$ $\mathrm{CO}_{2}$ for an additional 24 hours. At 48 hours after transfection, the supernatant was collected, spun at $500 \mathrm{~g}$ for 5 minutes at room temperature, filtered through a $0.45 \mu \mathrm{m}$ filter, and frozen at $-80^{\circ} \mathrm{C}$. The virus titers were evaluated using HEK293T-ACE2/TMPRSS2 cells at 10,000 cells per well on a Poly-L-Lysine-coated (5-10 $\mu \mathrm{g} / \mathrm{mL})$ 96-well plate using HI10 media (10\% heat-inactivated FBS, 1\% Pen/Strep), along with a virus dilution resulting in $>1000$ relative luciferase units (RLU) over control ( 1:100 virus stock dilution).

For the neutralization assay, 2.5-fold serial dilutions of the serum samples were incubated with diluted virus at a 1:1 ratio for 1 hour at $37^{\circ} \mathrm{C}$ before being transferred to plated HEK293-ACE2/TMPRSS2 cells and incubated for an additional 48 hours at $37^{\circ} \mathrm{C}$ and $5 \% \mathrm{CO}_{2}$. After 48 hours, cells were lysed, and Bright-Glo luciferase reagent (Promega, E2620) was added for 2 minutes before reading with a PerkinElmer Envision instrument.

\section{Statistics}

AUC values were tabulated for both the direct binding ELISA and the snELISA using R version 4.0.1 and $\mathrm{R}$ package pracma. For the snELISA, the ratios (normalized values) are used in the AUC calculations. To identify outliers, we calculated the distance of each point from the regression line using total least squares and labeled points with distances $>0.4$.

For the lentiviral pseudotyping assays, $50 \%$ inhibitory concentration or dilution $\left(\mathrm{IC}_{50}\right.$ or $\left.\mathrm{ID}_{50}\right)$ were calculated with nonlinear regression (log[inhibitor] versus normalized response - variable slope) using GraphPad Prism 8 (GraphPad Software Inc.). The "variable slope" option is a parameter selected in GraphPad Prism 8 for nonlinear regression that does not assume a standard slope of -1.0 with each dose-response curve but, instead, determines the slope of the curve based on the data generated.

For the extended direct binding dilution series, titres were calculated by taking the dilution of serum that produced $50 \%$ of the maximum response in the ELISA as determined by the nonlinear regression line (Sigmoidal, 4PL, $\mathrm{X}$ is $\log [$ dilution]) using GraphPad Prism 8.

The assay reproducibility was estimated across experiments by comparing the AUC values for those samples profiled across different batches.

CBS13 $(n=3) \mathrm{CV}$ for displacement was $5.1 \%$ and direct binding was 5.5\%; CBS16 $(n=3) \mathrm{CV}$ for displacement was $3.4 \%$ and $11.5 \%$ for binding; $\operatorname{CBS} 50(n=2) \mathrm{CV}$ for displacement was $9.9 \%$ and binding was $0.7 \%$.

When applicable, graphical data from experiments with 3 or more replicates are presented as mean \pm SEM.

\section{Study approval}

All samples were collected after Research Ethics Board (REB) review. The ELISAs were performed at the Lunenfeld-Tanenbaum Research Institute with Mount Sinai Hospital (MSH; Toronto, Ontario, Canada) REB approval (study no. 20-0078-E). External samples were transferred through Material Transfer Agreements. All research has been performed in accordance with relevant guidelines and regulations. All participants have provided informed consent. The samples were deidentified before transfer to the assay laboratory.

\section{Author contributions}

KTA and ACG designed the snELISA and the direct RBD antibody assay. ZL and JMR designed the protein expression, biotinylation, and purification procedures. RS and PST optimized the lentiviral pseudotyping assay. EJV, HW, MAD, APD, RCG, KAM, PB, and MO developed, performed and 
analyzed the PRNT and/or cytopathic effect-reduction neutralization assays. KTA and BR performed direct ELISA experiments. YD designed the VHH72hFc1X7 construct, expression, and purification procedures. JHW and MBR implemented the automated direct binding ELISA. KC helped coordinate the project. SJD provided samples, coordinated neutralization testing, and integrated PRNT and snELISA data. KTA and ACG analyzed the snELISA data. JLG, AJM, SM, MO, and SJD contributed essential patient samples. KTA, JMR, and ACG wrote the manuscript with input from all authors. The order of authors for the 2 co-first author was determined by the contribution of KTA in the overall study design, as well as data analysis and manuscript preparation.

\section{Acknowledgments}

We thank Janet McManus at Canadian Blood Services for her technical and logistical expertise and the Wadsworth Center Media and Tissue Culture Core. We thank Joan Wither for the lupus patient samples, and Jesse Bloom and Katharine Crawford for sharing protocols and reagents for the lentiviral S pseudotyping assay. The following reagent was produced by Florian Krammer's group under HHSN272201400008C and obtained through BEI Resources, NIAID, NIH: Spike Glycoprotein RBD from SARS-Related Coronavirus 2, WuhanHu-1, Recombinant from HEK293 Cells, NR-52306. We thank Matthew Stuible and Alex Pelletier (National Research Council; NRC) for VHH72hFc1X7 expression and purification, Joe Schrag (NRC) for SEC-UPLC/ MALS analysis, and members of our laboratories and the Network Biology Collaborative Centre for advice and technical help. Funding for the development of the assays in the Gingras lab was provided through generous donations from the Royal Bank of Canada (RBC), QuestCap, and the Krembil Foundation to the Sinai Health System Foundation. We also acknowledge an "Ontario Together" team grant from the Government of Ontario to JG, JMR, ACG, MO, and AM to develop serology and saliva assays. The equipment used is housed in the Network Biology Collaborative Centre at the Lunenfeld-Tanenbaum Research Institute, a facility supported by Canada Foundation for Innovation funding, by the Ontarian Government, and by Genome Canada and Ontario Genomics (OGI-139). We also acknowledge funding support from New York State Department of Health (to KAM). AJM, JMR, JLG, and ACG are supported by the Canadian Institutes of Health Research (CIHR 439999, FRN 162305, and FDN 143301, respectively). ACG is also supported by a Canada Research Chair, Tier 1, in Functional Proteomics. KTA is a recipient of an Ontario Graduate Scholarship.

Address correspondence to: James Rini, MaRS Centre, West Tower, Room 1614, 661 University Avenue, Toronto, Ontario M5G 1M1, Canada. Phone: 416.978.0742; Email: james.rini@utoronto.ca. Or to: AnneClaude Gingras, Mount Sinai Hospital, Lunenfeld-Tanenbaum Research Institute, 600 University Avenue, Room 992, Toronto, Ontario, M5G1X5, Canada. Phone: 416.450.5417; Email: gingras@lunenfeld.ca.

1. Hulswit RJ, de Haan CA, Bosch BJ. Coronavirus Spike Protein and Tropism Changes. Adv Virus Res. 2016;96:29-57.

2. Walls AC, Park YJ, Tortorici MA, Wall A, McGuire AT, Veesler D. Structure, Function, and Antigenicity of the SARS-CoV-2 Spike Glycoprotein. Cell. 2020;181(2):281-292.

3. Hoffmann M, et al. SARS-CoV-2 Cell Entry Depends on ACE2 and TMPRSS2 and Is Blocked by a Clinically Proven Protease Inhibitor. Cell. 2020;181(2):271-280.

4. Jiang S, Hillyer C, Du L. Neutralizing Antibodies against SARS-CoV-2 and Other Human Coronaviruses. Trends Immunol. 2020;41(5):355-359.

5. Corti D, Lanzavecchia A. Broadly neutralizing antiviral antibodies. Annu Rev Immunol. 2013;31:705-742.

6. Bloch EM, et al. Deployment of convalescent plasma for the prevention and treatment of COVID-19. J Clin Invest. 2020;130(6):2757-2765.

7. Mendoza EJ, Manguiat K, Wood H, Drebot M. Two Detailed Plaque Assay Protocols for the Quantification of Infectious SARS-CoV-2. Curr Protoc Microbiol. 2020;57(1):ecpmc105.

8. Crawford KHD, et al. Protocol and Reagents for Pseudotyping Lentiviral Particles with SARS-CoV-2 Spike Protein for Neutralization Assays. Viruses. 2020;12(5):E513.

9. Ferrara F, Temperton N. Pseudotype Neutralization Assays: From Laboratory Bench to Data Analysis. Methods Protoc. 2018;1(1):E8.

10. Amanat F, et al. A serological assay to detect SARS-CoV-2 seroconversion in humans. Nat Med. 2020;26(7):1033-1036.

11. Isho B, et al. Evidence for sustained mucosal and systemic antibody responses to SARS-CoV-2 antigens in COVID-19 patients. medRxiv. https://doi.org/10.1101/2020.08.01.20166553. Published August 29, 2020. Accessed September 2, 2020.

12. Crawford $\mathrm{KH}$, et al. Dynamics of neutralizing antibody titers in the months after SARS-CoV-2 infection. medRxiv. https://doi org/10.1101/2020.08.06.20169367. Published August 7, 2020. Accessed September 2, 2020.

13. Seow J, et al. Longitudinal evaluation and decline of antibody responses in SARS-CoV-2 infection. medRxiv. https://doi.org/1 0.1101/2020.07.09.20148429. Published July 11, 2020. Accessed September 2, 2020.

14. Yao XY, et al. Neutralizing and binding antibody kinetics of COVID-19 patients during hospital and convalescent phases. 
medRxiv. https://doi.org/10.1101/2020.07.18.20156810. Published July 21, 2020. Accessed September 2, 2020.

15. Wajnberg A, et al. SARS-CoV-2 infection induces robust, neutralizing antibody responses that are stable for at least 3 months. medRxiv. https://doi.org/10.1101/2020.07.14.20151126. Published July 17, 2020. Accessed September 2, 2020.

16. Tan CW, et al. A SARS-CoV-2 surrogate virus neutralization test based on antibody-mediated blockage of ACE2-spike protein-protein interaction [published ahead of print July 23, 2020]. Nat Biotechnol. https://doi.org/10.1038/s41587-020-0631-z.

17. Robbiani DF, et al. Convergent antibody responses to SARS-CoV-2 in convalescent individuals. Nature. 2020;584(7821):437-442.

18. Wrapp D, et al. Structural Basis for Potent Neutralization of Betacoronaviruses by Single-Domain Camelid Antibodies. Cell. 2020;181(5):1004-1015.e15.

19.Wan J, Xing S, Ding L, Wang Y, Zhu D, Rong B, Wang S, Chen K, He C, Yuan S, et al.: bioRxiv; 2020.

20. Long QX, et al. Clinical and immunological assessment of asymptomatic SARS-CoV-2 infections. Nat Med. 2020;26(8):1200-1204

21. Brouwer PJM, et al. Potent neutralizing antibodies from COVID-19 patients define multiple targets of vulnerability. Science. 2020;369(6504):643-650.

22. Chi X, et al. A neutralizing human antibody binds to the N-terminal domain of the Spike protein of SARS-CoV-2. Science. 2020;369(6504):650-655.

23. Wang C, et al. A human monoclonal antibody blocking SARS-CoV-2 infection. Nat Commun. 2020;11(1):2251.

24. Wu Y, et al. A noncompeting pair of human neutralizing antibodies block COVID-19 virus binding to its receptor ACE2. Science. 2020;368(6496):1274-1278.

25. Wan J, et al. Human-IgG-Neutralizing Monoclonal Antibodies Block the SARS-CoV-2 Infection. Cell Rep. 2020;32(3):107918

26. Darnell ME, Taylor DR. Evaluation of inactivation methods for severe acute respiratory syndrome coronavirus in noncellular blood products. Transfusion. 2006;46(10):1770-1777.

27. Li Z, Michael IP, Zhou D, Nagy A, Rini JM. Simple piggyBac transposon-based mammalian cell expression system for inducible protein production. Proc Natl Acad Sci U S A. 2013;110(13):5004-5009.

28. Tao Y, Strelkov SV, Mesyanzhinov VV, Rossmann MG. Structure of bacteriophage T4 fibritin: a segmented coiled coil and the role of the C-terminal domain. Structure. 1997;5(6):789-798.

29. Fairhead M, Howarth M. Site-specific biotinylation of purified proteins using BirA. Methods Mol Biol. 2015;1266:171-184.

30. Pallesen J, et al. Immunogenicity and structures of a rationally designed prefusion MERS-CoV spike antigen. Proc Natl Acad Sci U S A. 2017;114(35):E7348-E57.

31. ter Meulen J, et al. Human monoclonal antibody combination against SARS coronavirus: synergy and coverage of escape mutants. PLoS Med. 2006;3(7):e237.

32. Wang W, et al. Chromosomal transposition of PiggyBac in mouse embryonic stem cells. Proc Natl Acad Sci U S A. 2008;105(27):9290-9295.

33. Shi C, Shin YO, Hanson J, Cass B, Loewen MC, Durocher Y. Purification and characterization of a recombinant G-protein-coupled receptor, Saccharomyces cerevisiae Ste2p, transiently expressed in HEK293 EBNA1 cells. Biochemistry. 2005;44(48):15705-15714.

34. Poulain A, Perret S, Malenfant F, Mullick A, Massie B, Durocher Y. Rapid protein production from stable CHO cell pools using plasmid vector and the cumate gene-switch. J Biotechnol. 2017;255:16-27.

35. Stuible M, et al. Optimization of a high-cell-density polyethylenimine transfection method for rapid protein production in CHO-EBNA1 cells. J Biotechnol. 2018;281:39-47.

36. Wang S, Sakhatskyy P, Chou TH, Lu S. Assays for the assessment of neutralizing antibody activities against Severe Acute Respiratory Syndrome (SARS) associated coronavirus (SCV). J Immunol Methods. 2005;301(1-2):21-30.

37. Russell PK, Nisalak A, Sukhavachana P, Vivona S. A plaque reduction test for dengue virus neutralizing antibodies. J Immunol. 1967;99(2):285-290

38. Lindsey HS, Calisher CH, Mathews JH. Serum dilution neutralization test for California group virus identification and serology. J Clin Microbiol. 1976;4(6):503-510.

39. Calisher $\mathrm{CH}$, et al. Antigenic relationships between flaviviruses as determined by cross-neutralization tests with polyclonal antisera. J Gen Virol. 1989;70(Pt 1):37-43. 